\title{
Nivel de evidencia de la RICMA comparada con el JHSE y JHSA
}

\author{
R. Sánchez Rosales ${ }^{1}$, V. J. Hernández González², M. E. Pollwein². L. Reboso-Morales³, \\ Y. Martin Hidalgo?3. \\ 'Unidad de Cirugía de la Mano y Microcirugía, GECOT, San Cristóbal de La Laguna, Tenerife, España. ${ }^{2}$ Clínica de \\ Fisioterapia NOVA, S/C de Tenerife, España. ${ }^{3}$ Servicio de Cirugía Ortopédica y Traumatología, Hospital Universitario \\ de Nuestra Señora de Candelaria HUNSC. Universidad de La Laguna. San Cristóbal de La Laguna, Tenerife, España.
}

\begin{abstract}
Resumen: Objetivo: El propósito del presente trabajo fue evaluar el nivel de evidencia científica de artículos de investigación clínica publicados en las Revista Ineroamericana de Cirugía de la Mano (RICMA) y compararlo con las revistas Europea (JHSE) y Americana (JHSA). Material y método: Un total de 932 trabajos de investigación clínicos publicados entre 2005 y 2009 (RICMA 60, JHSE, JHSA 46I y 4II) fueron revisados. Dos observadores independientes clasificaron el nivel de evidencia basado en la Clasificación Internacional de Oxford, siendo 5 el nivel más bajo y I el nivel más alto de evidencia científica. Las frecuencias observadas de nivel de evidencia para cada revista científica fueron comparadas con las frecuencias esperadas mediante un test de chi- cuadrado $\left(X^{2}\right)$ para variables categóricas con un nivel de significancia del 0.05 .

Resultados: Más del $80 \%$ de los trabajos publicados en la RICMA y el JHSE , y el $67.6 \%$ en el JHSA presentaron un nivel IV de evidencia. Trabajos de nivel I o II no fueron publicados en la RICMA, comparado con el JHSE ( $0.9 \%$ nivel I, $5 \%$ nivel II), y con el JHSA ( $8.3 \%$ nivel I, $10 \%$ nivel II). El porcentaje de artículos con nivel III publicados en la RICMA (I6,7\%) fue mayor al observado en el JHSE (II.I\%) y en el JHSA (14.1\%). Todos los resultados fueron estadísticamente significativos $\left(X^{2}=63.95 ; p<0.001\right)$.

Conclusiones: El nivel de evidencia de las publicaciones en investigación clínica en cirugía de la mano depende del tipo de revista, siendo el mayor nivel de evidencia en aquellos trabajos publicados en el JHSA, seguido del JHSE y finalmente la RICMA.
\end{abstract}

Palabras claves: Nivel de evidencia científica, Cirugía de mano, Investigación clínica.

\section{Level of evidence of the clinical research published in the lbero-ameri- can Journal of Hand Surgery (RICMA) compared to the JHSE and JHSA}

\begin{abstract}
Objective:The purpose of this study was to assess the level of evidence of the clinical research papers published in the lberoamerican (RICMA), the European (JHSE) and American (JHSA) Journals of Hand Surgery.

Methods: A total of 932 clinical research papers published between 2005 and 2009 (RICMA 60, JHSE 46I, and JHSA 4II) were reviewed. Two independent observers classified the level of evidence based on the Oxford International Classification, 5 being the lowest level and I the highest level. The observed frequencies of the level of evidence for each journal were compared with the expected frequencies by a chi-square $\left(X^{2}\right)$ test for categorical variables with a significance level of 0.05 .

Results: More than $80 \%$ of the papers in RICMA and JHSE and a $67.6 \%$ in the JHSA presented a level of 4 . No level I or 2 studies were published in RICMA, compared to JHSE (0.9\% level I and 5.0\% level 2) and JHSA ( $8.3 \%$ level I and I0\% level 2). The percentage of papers with level 3 published in RICMA (16.7\%) was higher compared to the JHSE (II.I\%) and the JHSA (I4.1\%).All the results were statistically significant $\left(X^{2}=63.945 ; p<0.00 I\right)$.

Conclusion:The level of evidencie in clinical resarch related to hand surgery depend on the type of journal, being the higuest level of evidence in papers published in the JHSA, followed by the JHSE and finally, the RICMA.
\end{abstract}

Key words: Level of evidence, Hand surgery, Clinical research.

Desde que fue descrito el primer sistema de clasificación del nivel de evidencia científica de los artículos de investigación clínica', la Medicina Basada en la Evidencia ha llegado a ser una importante parte de la práctica clínica. Los cirujanos de mano deberían entender el nivel de evidencia para llegar a ser conocedores de la veracidad y la utilidad de los datos contenidos en los artículos consultados. Pocas investigaciones se han hecho para analizar el nivel de evidencia cientíica en revistas relacionadas con cirugía de mano en relación a otros campos de investigación clínica afines, como en revistas de cirugía ortopédica 2,3 y cirugía plástica ${ }^{4}$. Sólo una revista específica de cirugía de mano ha sido analizada para ver su nivel de evidencia en un
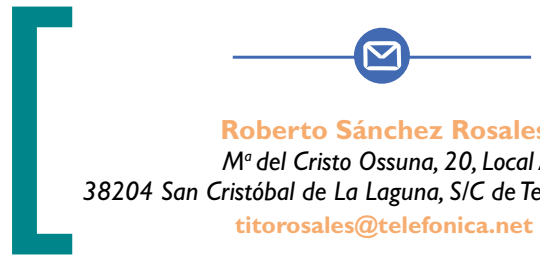

Roberto Sánchez Rosales

$M^{a}$ del Cristo Ossuna, 20, Local A

38204 San Cristóbal de La Laguna, S/C de Tenerife, España titorosales@telefonica.net 
período de seis meses, y ha sido comparada con otras de publicaciones ortopédicas ${ }^{3}$. Hasta nuestro conocimiento, no existen trabajos previos publicados en lengua castellana que comparen el nivel de evidencia científica de la RICMA con otras revistas científicas del campo de la cirugía de la mano. El propósito de este trabajo fue evaluar el nivel de evidencia científica de los artículos de investigación clínica publicados entre 2005 y 2009 en la Revista Ibero-Americana de Cirugía de Mano (RICMA), considerada como la revista y órgano de expresión de la Sociedad Española de Cirugía de Mano y de las que son Sociedades Científicas Afiliadas, la Sociedad Portuguesa de Cirugía de Mano y la mayor parte de las sociedades de Cirugía de Mano de Latino América y compararlo con la Revista Europea de Cirugía de Mano (JHSE) y la Americana (JHSA), como las revistas oficiales de la Federación de las Sociedades Europeas de Cirugía de Mano (FESSH) y la Sociedad Americana de Cirugía de Mano (ASSH).

Los investigadores establecieron como hipótesis nula $(\mathrm{Ho})$ que la variable "nivel de evidencia científica" era independiente de la variable "tipo de revista"

\section{MATERIALY MÉTODO}

\section{Población de estudio}

Criterios de inclusión: Todos los artículos de investigación clínica que fueron publicados entre Enero de 2005 y Diciembre de 2009 en la Revista Ibero-Americana de Cirugía de Mano (RICMA), la Revista Europea de Cirugía de la Mano (JHSE) y la Revista Americana de Cirugía de la Mano (JHSA).

Criterios de exclusión: Estudios animales, anatómicos y de cadáver, estudios básicos de ciencias, cursos de instrucción, suplementos de resúmenes, publicaciones cortas, cartas al editor y artículos de revisión no fueron considerados factibles para el estudio.

Por esta razón, un total de 932 artículos de investigación clínica siguieron los criterios de inclusión y exclusión (RICMA 60, JHSE 46 I y JHSA 4II).

\section{Evaluación del Nivel de Evidencia}

Los artículos que siguieron los criterios de inclusión y exclusión fueron asignados de forma aleatoria a dos observadores independientes (YMH, LRM), con una larga experiencia clínica en cirugía de la mano y bien familiarizados con la Medicina Basada en la Evidencia. Un número igual aproximado de artículos de cada una de las revistas fue evaluado por cada observador.
El nivel de evidencia científica para cada artículo fue evaluado en base a "Niveles de Evidencia científica - Centro de Oxford para la Medicina Basada en la Evidencia"'. La valoración fue a ciegas en relación a cualquier información previa del nivel de evidencia de los artículos analizados (e.g.: "nivel de evidencia y tipo de estudio", el cual es incluido en la JHSA con el resumen de cada artículo desde 2006). Los artículos fueron catalogados de acuerdo a su nivel de evidencia siendo el nivel I (mayor nivel de evidencia, e.g: revisiones sistemáticas (SR), meta-análisis (MA), y estudios individuales controlados y aleatorizados con estrechos intervalos de confianza), y el nivel 5 (menor nivel de evidencia, e.g.: opinión de experto). La evaluación de los sub-grupos de nivel de evidencia, así como los tipos de artículos científicos no fueron realizados por los observadores. Tabla I.

\section{Análisis de la fiabilidad}

Antes de empezar el estudio, la fiabilidad de la evaluación fue hecha en base al análisis del error intra-observador e inter-observador. Una muestra aleatoria de 30 artículos de investigación clínica, de un total de 872 artículos, publicados en Inglés (46I de JHSE, y 4 II de $J H S A$ ), fueron evaluados por dos observadores independientes asignados para el estudio. Después de 15 días, se hizo una segunda evaluación con el orden de los artículos cambiado. Ningún artículo de la RICMA fue incluido en la muestra de estudio para el análisis de la fiabilidad. Esto se hizo para evitar un sesgo de información ${ }^{6}$, porque las diferentes lenguas presentes en la RICMA (Español y Portugués), podría aumentar la fiabilidad intra-observador. La fiabilidad intra e interobservador fue estudiada usando el test Coeficiente Kappa con un nivel de significancia de 0.05 .

\section{Análisis estadístico}

Para la evaluación de los resultados, el número de artículos clasificados para cada nivel de evidencia fue expresado como un porcentaje del total de artículos que cumplieron con los criterios de inclusión y exclusión durante el período de tiempo estudiado. Las frecuencias observadas para cada nivel de evidencia de cada revista fueron comparadas con las frecuencias esperadas usando un test chi-cuadrado $\left(x^{2}\right)$ para variables categóricas con un nivel de significancia de 0.05.

\section{RESULTADOS}

El análisis inter-observador mostró un Kappa de 0.617, con un error asintótico estándar (SE) de 0.1 I 17. El análisis intra-observador presentó un Kappa de 0.66 (SE 


\section{TABla I - Nivel de eVIDencia Y TiPO de estudio.}

\begin{tabular}{|c|c|c|c|c|c|}
\hline & $\begin{array}{l}\text { Tratamiento, } \\
\text { prevención, } \\
\text { etiología, } \\
\text { daño }\end{array}$ & $\begin{array}{c}\text { Pronóstico } \\
\text { Investigar el efecto } \\
\text { de la característica } \\
\text { del paciente en } \\
\text { el resultado de la } \\
\text { enfermedad }\end{array}$ & $\begin{array}{l}\text { Diagnóstico } \\
\text { Investigar un } \\
\text { test diagnóstico. } \\
\text { ¿Es este test } \\
\text { diagnóstico exacto? }\end{array}$ & $\begin{array}{l}\text { Diagnóstico } \\
\text { diferencial, estudios } \\
\text { de prevalencia }\end{array}$ & $\begin{array}{l}\text { Estudios económicos } \\
\text { y Análisis de decisión }\end{array}$ \\
\hline \multirow[t]{2}{*}{ Nivel I } & $\begin{array}{l}\text { Revisión sistemática de ensa- } \\
\text { yos aleatorizados (ECA) }\end{array}$ & $\begin{array}{l}\text { Revisión sistemática de es- } \\
\text { tudios de cohortes }\end{array}$ & $\begin{array}{l}\text { Revisión sistemática } \\
\text { de estudios diagnósti- } \\
\text { cos de nivel I }\end{array}$ & $\begin{array}{l}\text { Revisión sistemática de es- } \\
\text { tudios de cohortes clásico o } \\
\text { prospectivos }\end{array}$ & $\begin{array}{l}\text { Revisión sistemática de estu- } \\
\text { dios económicos de nivel I }\end{array}$ \\
\hline & $\begin{array}{l}\text { ECA de alta calidad } \\
\text { (e.g. seguimiento superior al } \\
80 \% \text {, con un intervalo de con- } \\
\text { flanza estrecho) }\end{array}$ & $\begin{array}{l}\text { Estudio de cohortes indi- } \\
\text { vidual con un seguimiento } \\
\text { superior al } 80 \% \text {, con todos } \\
\text { los pacientes listados al } \\
\text { mismo tiempo }\end{array}$ & $\begin{array}{l}\text { Estudios diagnósticos de } \\
\text { nivel I o estudios de va- } \\
\text { lidación que prueben la } \\
\text { calidad de un test diagnós- } \\
\text { tico espećíico, previamente } \\
\text { desarrollado, en series de } \\
\text { pacientes consecutivos con } \\
\text { referencia a "gold standard" }\end{array}$ & $\begin{array}{l}\text { Estudios prospectivos o co- } \\
\text { hortes clásico con buen } \\
\text { seguimiento (>80\%) }\end{array}$ & $\begin{array}{l}\text { Estudios de nivel I (Análisis } \\
\text { basado en costes clínicamen- } \\
\text { te sensibles o alternativos, va- } \\
\text { lores obtenidos de muchos } \\
\text { estudios, e incluyendo análi- } \\
\text { sis de sensibilidad de diferen- } \\
\text { tes maneras }\end{array}$ \\
\hline \multirow[t]{3}{*}{ Nivel II } & $\begin{array}{l}\text { Revisión sistemática de estu- } \\
\text { dios de cohortes }\end{array}$ & $\begin{array}{l}\text { Revisión sistemática de } \\
\text { cualquier estudio histórico } \\
\text { de cohorte o de grupos } \\
\text { controles no tratados } \\
\text { (brazo control) en RCTs }\end{array}$ & $\begin{array}{l}\text { Revisión sistemática de } \\
\text { estudios diagnósticos de } \\
\text { nivel } 2\end{array}$ & $\begin{array}{l}\text { Revisión sistemática de estu- } \\
\text { dios de nivel } 2\end{array}$ & $\begin{array}{l}\text { Revisión sistemática de estu- } \\
\text { dios de nivel } 2\end{array}$ \\
\hline & $\begin{array}{l}\text { ECA de menor calidad } \\
\text { (e.g. seguimiento inferior al } \\
80 \% \text {, con un intervalo de con- } \\
\text { flanza amplio) }\end{array}$ & $\begin{array}{l}\text { Estudio histórico de } \\
\text { cohortes (retrospectivo) } \\
\text { o brazo de control de un } \\
\text { RCT }\end{array}$ & $\begin{array}{l}\text { Estudios de diagnóstico de } \\
\text { nivel } 2 \text { o estudios explo- } \\
\text { ratorios que, a través de } \\
\text { una regresión logística, de- } \\
\text { terminan factores significa- } \\
\text { tivos (e.g.g. usando regresión } \\
\text { de análisis) }\end{array}$ & $\begin{array}{l}\text { Estudios de nivel } 2 \text { (Estudios } \\
\text { retrospectivos o de cohor- } \\
\text { tes histórico, o con un se- } \\
\text { guimiento inferior al <80\%) }\end{array}$ & $\begin{array}{l}\text { Estudios de nivel } 2 \text { (Análisis } \\
\text { basados en costes clínicamen- } \\
\text { te sensibles o alternativas de } \\
\text { estudios limitados, e inclu- } \\
\text { yendo análisis de sensibilidad } \\
\text { de diferentes maneras }\end{array}$ \\
\hline & $\begin{array}{l}\text { Estudio de cohortes indi- } \\
\text { vidual, incluye estudios de } \\
\text { cohortes apareados (Estudios } \\
\text { prospectivos comparativos) } \\
\text { Estudios ecológicos }\end{array}$ & & & Estudios ecológicos & \\
\hline \multirow[t]{2}{*}{ Nivel III } & $\begin{array}{l}\text { Revisión sistemática de estu- } \\
\text { dios casos-control }\end{array}$ & & $\begin{array}{l}\text { Revisión sistemática de es- } \\
\text { tudios de nivel } 3\end{array}$ & $\begin{array}{l}\text { Revisión sistemática de estu- } \\
\text { dios de nivel } 3\end{array}$ & $\begin{array}{l}\text { Revisión sistemática de estu- } \\
\text { dios de nivel } 3\end{array}$ \\
\hline & $\begin{array}{l}\text { Estudio caso-control indi- } \\
\text { vidual }\end{array}$ & & $\begin{array}{l}\text { Estudios diagnósticos de } \\
\text { nivel } 3 \text { o estudios en pa- } \\
\text { cientes no consecutivos y } \\
\text { sin referencias consistentes } \\
\text { de "gold standards". }\end{array}$ & $\begin{array}{l}\text { Estudios de nivel } 3 \text { (cohortes } \\
\text { no consecutivos } 0 \text { pobla- } \\
\text { ción muy limitada) }\end{array}$ & $\begin{array}{l}\text { Estudios de nivel } 3 \text { (análisis } \\
\text { basado en pocas alternativas } \\
\text { o costes, poca calidad de } \\
\text { estimación de datos, pero } \\
\text { incluyendo análisis de la sen- } \\
\text { sibilidad) }\end{array}$ \\
\hline \multirow[t]{2}{*}{ Nivel IV } & Serie de casos & Serie de casos & Estudio de caso - control & Serie de casos & No análisis de sensibilidad \\
\hline & $\begin{array}{l}\text { Estudios Cohortes y caso- } \\
\text { control de poca calidad* }\end{array}$ & $\begin{array}{l}\text { Estudios Cohortes y caso- } \\
\text { control de poca calidad* }\end{array}$ & $\begin{array}{l}\text { Estándares de referencia } \\
\text { pobres o no independien- } \\
\text { tes }\end{array}$ & & \\
\hline NivelV & Opinión de experto & Opinión de experto & Opinión de experto & Opinión de experto & Opinión de experto \\
\hline
\end{tabular}

Generalmente, una revisión sistemática (RS) es mejor que cualquier estudio individual. Un estudio experimental (e.g.: ensayo controlado aleatorizado de buena calidad) es normalmente mejor que cualquier estudio observacional. Para estudios observacionales: un estudio cohorte es mejor que cualquier estudio casos-control. Un estudio de casos-control es generalmente mejor que cualquier estudio de 
serie de casos. *Como "estudios cohortes de baja calidad" nos referimos a aquellos cohortes que son deficientes a la hora de definir claramente los grupos comparados y/o deficientes a la hora de medir exposiciones y resultados (preferiblemente a ciegas) de la misma objetiva manera en ambos grupos (expuestos y no expuestos) ylo deficientes a la hora de identificar las variables de confusión ylo con un seguimiento muy pobre. Lo mismo para "estudios casos-control de baja calidad" a excepción de que en los diseños clínicos de casos-control los pacientes entran en el estudio en base a la variable "resultado". Así los "casos" (e.g.: fracaso de reimplante) son comparados con aquellos que no presentan la variable resultado (e.g.: reimplante exitoso) llamados "control" y por tanto no tenemos "expuestos y no expuestos) ni tampoco tenemos seguimiento longitudinal. Estudios ecológicos y Económicos/ análisis de desición son muy poco frecuentes en cirugía de la mano. Esta tabla ha sido adaptada de material publicado por el Centro de medicina Basada en la Evidencia, Oxford, Inglaterra. Marzo de 2009.

0.1 |4) para el observador I, y un Kappa de 0.75। (SE $0.103)$ para el observador 2. Todos los valores Kappa fueron significativos $(p<0.001$ ). Más del $80 \%$ de los artículos publicados en RICMA y la JHSE; y un 67.6\% en la JHSA presentaron un nivel 4 de evidencia. Ningún artículo con nivel I y || de evidencia fue publicado en la RICMA comparado con la JHSE $(0.9 \%$ de nivel I y $5.0 \%$ de nivel II) y la JHSA (8.3\% de nivel I y $10 \%$ de nivel II). El porcentaje de artículos con nivel III, publicados en la RICMA (16.7\%) fue mayor comparado con la JHSE (II.1\%) y la JHSA (14.1\%) (2) Tabla II. Todos los resultados fueron estadísticamente significativos $\left(x^{2}=63.945 ; p<0.001\right)$ y la Hipótesis nula (Ho) fue rechazada.

\section{DISCUSIÓN}

Los resultados de este artículo han demostrado con un nivel de fiabilidad bueno-excelente que la variable "nivel de evidencia científica" es dependiente de la variable "tipo de revista".
El uso de Kappa es importante, ya que nos permite conocer el grado de acuerdo intra-observador e interobservadores teniendo en cuenta el azar. Un coeficiente Kappa estadísticamente significativo indica que el acuerdo es diferente a cero (acuerdo nulo). Sin embargo, la interpretación de valores Kappa obtenidos es subjetivo, y han sido propuestas diferentes clasificaciones o guías de interpretación del coeficiente Kappa para el análisis de fiabilidad. En este artículo, el nivel de acuerdo de análisis del inter-observador y el intra-observador ha mostrado un valor Kappa comprendido desde 0.617 a 0.751 , que puede ser considerado que tiene un nivel de fiabilidad de buena a excelente ${ }^{7,8}$, en la evaluación del nivel de evidencia y el tipo de revista.

Resultados similares han sido publicados antes. Obremskey y cols. ${ }^{3}$, en el estudio del nivel de evidencia de revistas de ortopedia, han publicado valores Kappa de 0.62 para acuerdos inter-observadores entre revisores con poca experiencia, y valores Kappa de 0.75

\section{TABLA II - TABULACIÓN CRUZADA DE "TIPO DE REVISTA" Y "NIVEL DE EVIDENCIA"}

\begin{tabular}{|c|c|c|c|c|c|c|}
\hline \multirow[t]{2}{*}{ REVISTA } & \multicolumn{5}{|c|}{ NIVEL DE EVIDENCIA } & \multirow{2}{*}{$\begin{array}{c}\text { NUMERO } \\
\text { COMPLETO } \\
\text { DETRABAJOS }\end{array}$} \\
\hline & I & II & III & IV & $\mathbf{v}$ & \\
\hline \multirow{3}{*}{$\begin{array}{l}\text { RICMA } \\
\text { (IC 95\%) }\end{array}$} & $0.0 \%$ & $0.0 \%$ & $16.7 \%$ & $80.0 \%$ & $3.3 \%$ & \\
\hline & (N.A.) & (N.A.) & $(7.3 ; 26.1)$ & $(69.9 ; 90.1)$ & (N.A.) & \\
\hline & 0 & 0 & 10 & 48 & 2 & 60 \\
\hline \multirow{3}{*}{$\begin{array}{l}\text { JHSE } \\
\text { (IC 95\%) }\end{array}$} & $0.9 \%$ & $5.0 \%$ & $11.1 \%$ & $82.4 \%$ & $0.7 \%$ & \\
\hline & (N.A.) & $(3.02 ; 6.9)$ & & & (N.A.) & \\
\hline & 4 & 23 & 51 & 380 & 3 & 461 \\
\hline \multirow{3}{*}{$\begin{array}{l}\text { JHSA } \\
\text { (IC 95\%) }\end{array}$} & $8.3 \%$ & $10 \%$ & $14.1 \%$ & $67.6 \%$ & $0 \%$ & \\
\hline & $(5.6 ; 10.9)$ & $(7.1 ; 12.9)$ & $(10.7 ; 17.4)$ & $(63.1 ; 72.1)$ & (N.A.) & \\
\hline & 34 & 41 & 58 & 278 & 0 & 411 \\
\hline
\end{tabular}

RICMA: Revista Iberoamericana de Cirugía de la Mano / JHSE: Journal of Hand Surgery European Volume / JHSA: Journal of Hand Surgery American Volume / IC: intervalo de confianza / N.A. no aplicable: (cuando el numero de proporción observado fue menor de 5/n) 
para inter-observadores experimentados. Ningún análisis intra-observador fue publicado por estos autores.

No muchos artículos han estudiado el nivel de evidencia científica en revistas de cirugía de la mano o en campos similares de investigación, como revistas de cirugía ortopédica y plástica. Sinno y cols. ${ }^{4}$, revisaron 726 artículos de 6 revistas diferentes de cirugía plástica y el nivel de evidencia fue evaluado usando una clasificación basada en el Centro para el Nivel de Evidencia de Oxford (CEBM). En Hanzlik y cols. ${ }^{2}$ fueron evaluados 55I artículos del Volumen Americano del JBJS de los años 1975 ( 134 artículos), 1985 (I 23 artículos), 1995 (I 20 artículos) y 2005 (I 74 artículos). El nivel de evidencia fue evaluado siguiendo una clasificación incluida en la guía de autores (sistema de clasificación JBJSA), la cual es muy similar a la desarrollada por la CEBM, con el fin de demostrar la tendencia del nivel de evidencia durante 30 años. Obremskey y cols. ${ }^{3}$ revisaron 382 artículos de investigación clínica de nueve revistas diferentes con el fin de evaluar el nivel de evidencia científica en revistas de cirugía ortopédica. En el presente trabajo fueron revisados 932 artículos de tres revistas específicas de cirugía de la mano, lo cual supone la población más amplia estudiada hasta ahora, para evaluar el nivel de evidencia científica.

Los resultados de este trabajo demuestran que la mayoría de los artículos clínicos publicados en cirugía de la mano tienen muy poco nivel de evidencia científica (80\% nivel IV en la JHSE o RICMA y $67.6 \%$ en la JHSA). La mayoría de los artículos fueron serie de casos y menos frecuentemente estudios cohortes y casos-control de pobre calidad comparado con revistas científicas de cirugía ortopédica (48\% estudios de nivel IV) ${ }^{2}$, plástica (40\% estudios de nivel IV) ${ }^{4}$ y de oftalmología ( $58 \%$ estudios de nivel IV) ${ }^{9}$. Sin embargo, otras revistas de cirugía, como las relativas a otorrinolaringología, presentan un porcentaje similar a la JHSE y RICMA ( $80 \%$ estudios de nivel IV) ${ }^{10}$. El porcentaje de artículos de nivel IV en la JHSA fue menor, comparado con el resto de revistas de cirugía de mano investigadas, y fue muy similar al del trabajo publicado por Obremskey y cols. ${ }^{3}$, donde se notifica la existencia de un $68.8 \%$ de artículos de nivel IV, en una revisión de 32 artículos publicados en la JHSA de Enero a Junio de 2003.

El porcentaje de artículos con un alto nivel de evidencia científica (nivel I y II) fue mayor en la JHSA (8.3\% nivel I y $10 \%$ nivel II), comparado con la RICMA (0\%) y la JHSE (0.9\% nivel I y $5 \%$ nivel II). Mientras que si se compara con otras revistas, hubo un $21 \%$ de nivel I y un 15\% de nivel II de evidencia en revistas de cirugía ortopédica ${ }^{2}, 3 \%$ de nivel I y $16 \%$ de nivel II en cirugía plástica ${ }^{4}, 18 \%$ de nivel I y $8 \%$ de nivel II en revistas de oftalmología 9 , y $7 \%$ de niveles I y II en otorrinolaringología 10 .

El porcentaje de artículos con nivel III (mayoritariamente estudios de casos-control y cohortes no consecutivos o con poblaciones muy limitadas) publicados en la RICMA (16.7\%) fue mayor comparado con la JHSE (I I\%) y la JHSA ( 14\%); y similar a otras revistas: $16 \%$ en revistas de cirugía ortopédica ${ }^{2}$, 16\% en otorrinolaringología ${ }^{10}$, y $16 \%$ en oftalmología?.

Algunos autores han criticado el escaso número de artículos de alto nivel de evidencia en cirugía". Aun así, las críticas puede que parezcan exageradas si se tiene en cuenta que un ensayo clínico aleatorizado quirúrgico es diferente de un ensayo que compara un medicamento con un placebo. Los procedimientos quirúrgicos son invasivos; es difícil aleatorizar pacientes, el enmascaramiento es un problema en un ensayo quirúrgico, y ambos son muy caros. Si no se tiene estudios aleatorios de alta calidad, no se puede tener una revisión sistemática que sintetice la evidencia anteriormente descrita.

Ningún análisis de tendencia es una limitación de este estudio, y dicha información debería ser el propósito de futuros estudios, en el sentido de entender cómo la evidencia cientíica publicada en revistas de cirugía de la mano ha cambiado, y cómo la relación entre cambios en el nivel de evidencia y cambios en el índice de impacto ha variado también con el tiempo.

Después de revisar una enorme cantidad de artículos publicados en revistas de diferentes partes del mundo, otras cuestiones afloran, tales como si las diferencias encontradas son un reflejo de diferentes prioridades regionales, o de qué manera las inversiones utilizadas para la investigación tienen un impacto en los resultados, e incluso si diferentes países son los principales contribuidores en estudios de alto nivel.

\section{CONCLUSIONES}

El nivel de evidencia científica en cirugía de la mano es dependiente del tipo de revista; siendo los artículos de mayor nivel de evidencia los publicados en la JHSA, seguido por la JHSE y finalmente la RICMA. Conocer el estado del nivel de evidencia publicado en cirugía 
de la mano, es el punto de partida para enfocar los cambios de mejora de la calidad de nuestros estudios clínicos. Así, la existencia de un 16\% de artículos de nivel 3 de evidencia en la RICMA, superior a las otras revistas, puede ser considerado ese "punto de partida" hacia la mejora de la calidad de los artículos que se publican en nuestra revista oficial de la Sociedad Española de Cirugía de la Mano.

\section{CONFLICTOS DE INTERESES}

Los autores declaran no tener conflictos de intereses.

\section{BIBLIOGRAFÍA}

I. Sackett DL. Rules of evidence and clinical recommendations on the use of antithrombotic agents. Chest. 1986;89(2 Suppl):25-35.

2. Hanzlik S, Mahabir RC, Baynosa RC, Khiabani KT. Levels of evidence in research published in the journal of bone and joint surgery (American volume) over the last thirty years.J Bone Joint Surg. 2009;9|A:425-8.
3. Obremskey WT, Pappas N, Attallah-Wasif E, Tornetta $\mathrm{P}$, Bhandari M. Level of evidence in orthopedic journals.J Bone Joint Surg. 2005;87A:2632-8.

4. Sinno H, Neel OF, Lutty J, Bartlett G, Gilardino M. Level of evidence in plastic surgery research. Plast Reconstr Surg. 201 I; 127:974-80.

5. Oxford Center for Evidence-based Medicine:Levels of Evidence. Disponible en: http://www.cebm.net/ oxford-centre-evidence-based-medicine-levels-evidence-march-2009/.

6. Page RM, Cole GE,TimmreckTC. Basic Epidemiological Methods and Biostatistics. A practical guidebook. Boston: Jones and Bartlett publishers; 1995.

7. Landis JR, Koch GG. The measurements of observer agreement for categorical data. Biometrics. 1977;33:159-74.

8. Silman AJ. Epidemiological Studies: a practical guide. New York: Cambridge University Press; 1995.

9. LaiTY, Leung GM, WongWW, Lam RF, Cheng AC, Lam DS. How evidence-based are publications in clinical ophthalmic journals? Invest Ophthalmol Vis Sci. 2006:47:1831-8.

10. Bentsianov BL, Boruk M, Rosendfield RM. Evidencebased medicine in otolaryngology journals. Otolaryngol Head Neck Surg.

I I. Horton R. Surgical research or comic opera: questions, but few answers. Lancet. 1996;347:984-5. 